\title{
Investigating a Coupled Hybrid System of Nonlinear Fractional Differential Equations
}

\author{
Wiyada Kumam $\mathbb{D}^{1},{ }^{1}$ Mian Bahadur Zada $\mathbb{D}^{1},{ }^{2}$ Kamal Shah $\mathbb{D}^{2},{ }^{2}$ and Rahmat Ali Khan ${ }^{2}{ }^{2}$ \\ ${ }^{1}$ Program in Applied Statistics, Department of Mathematics and Computer Science, Faculty of Science and Technology, \\ Rajamangala University of Technology Thanyaburi, Thanyaburi, Pathum Thani 12110, Thailand \\ ${ }^{2}$ Department of Mathematics, University of Malakand, Chakdara, Khyber Pakhtunkhwa, Pakistan \\ Correspondence should be addressed to Wiyada Kumam; wiyada.kum@rmutt.ac.th
}

Received 8 December 2017; Accepted 30 January 2018; Published 20 March 2018

Academic Editor: Youssef N. Raffoul

Copyright (c) 2018 Wiyada Kumam et al. This is an open access article distributed under the Creative Commons Attribution License, which permits unrestricted use, distribution, and reproduction in any medium, provided the original work is properly cited.

We study sufficient conditions for existence of solutions to the coupled systems of higher order hybrid fractional differential equations with three-point boundary conditions. For this motive, we apply the coupled fixed point theorem of Krasnoselskii type to form adequate conditions for existence of solutions to the proposed system. We finish the paper with suitable illustrative example.

\section{Introduction}

Fractional calculus is found to be more practical and effective than the classical calculus in the mathematical modeling of several phenomena. Fractional differential equations are very important and significant part of the mathematics and have various applications in viscoelasticity, electroanalytical chemistry, and many physical problems [1-6]. A systematic presentation of the applications of fractional differential equations can be found in the book of Balachandran and Park [7]. In recent years, many works have been devoted to the study of the mathematical aspects of fractional order differential equations [8-12]. There are numerous advanced and efficient methods, which have been focusing on the existence of solution to fractional differential equations. One of the powerful tools for obtaining the existence of solutions to such equations is the fixed point methods. Many authors use fixed point theorems to prove the existence and uniqueness of solution to nonlinear fractional differential equations; see, for example, [13-17].

On the other hand, the study for coupled systems of fractional differential equations is also important as such systems occur in various problems of applied nature, for instance, [18-25]. Additionally, fixed point theory can be used to develop the existence theory for the coupled systems of fractional hybrid differential equations $[13,16,17]$. Bashiri et al. [17] discussed the existence of solution to the following system of fractional hybrid differential equations of order $p \in(0,1)$ :

$$
\begin{aligned}
D^{p}[x(t)-f(t, x(t))] & =g\left(t, y(t), I^{\alpha} y(t)\right), \\
\text { a.e. } t \in[0, T], T>0, & \\
D^{p}[y(t)-f(t, y(t))] & =g\left(t, x(t), I^{\alpha} x(t)\right), \\
\text { a.e. } t \in[0, T], T>0, & \\
x(0) & =0, \\
y(0) & =0,
\end{aligned}
$$

where $\alpha>0$, and the functions $f:[0, T] \times \mathbb{R} \rightarrow \mathbb{R}, f(0,0)=$ 0 and $g:[0, T] \times \mathbb{R} \times \mathbb{R} \rightarrow \mathbb{R}$ satisfy certain conditions. $D^{p}$ is the R-L fractional derivative of order $p$.

Recently, the existence of solutions for fractional differential equations involving the Caputo fractional derivative was studied in [13, 26-28]. Motivated by the work of Bashiri et al. [17], in this paper we are concerned with the existence of solutions to three-point boundary value problem for a 
coupled system of hybrid fractional differential equations of order $p \in[n-1, n)$ given by

$$
\begin{aligned}
& D^{p}[x(t)-f(t, x(t))]=g\left(t, y(t), I^{\alpha} y(t)\right), \\
& D^{p}[y(t)-f(t, y(t))]=g\left(t, x(t), I^{\alpha} x(t)\right), \\
& \text { a.e. } t \in[0,1], \text { a.e. } t \in[0,1], \\
& x(0)=\delta_{1} x\left(\eta_{1}\right), \\
& y(0)=\delta_{1} y\left(\eta_{1}\right), \\
& x(1)=\delta_{2} x\left(\eta_{2}\right), \\
& y(1)=\delta_{2} y\left(\eta_{2}\right), \\
& x^{(i)}(0)=y^{(i)}(0)=0, \\
& \quad \text { for } i=1,2,3, \ldots,(n-2),
\end{aligned}
$$

where $\alpha>0,0<\eta_{1}<\eta_{2}<1, f:[0,1] \times \mathbb{R} \rightarrow \mathbb{R}$, $f(0, x(0))=0$, and $g:[0,1] \times \mathbb{R} \times \mathbb{R} \rightarrow \mathbb{R} . D^{p}$ is the Caputo fractional derivative of order $p$. Moreover, an example is given to illustrate the validity of the existence result.

\section{Preliminaries}

Throughout this manuscript $\Phi=\left\{\varphi: \mathbb{R}^{+} \rightarrow\right.$ $\mathbb{R}^{+}$such that $\varphi(r)<r$ for $r>0$ and $\left.\varphi(0)=0\right\}$, $C([0, T] \times \mathbb{R}, \mathbb{R})$ denote the class of continuous functions $f:[0, T] \times \mathbb{R} \rightarrow \mathbb{R}$, and $C([0, T] \times \mathbb{R} \times \mathbb{R}, \mathbb{R})$ denote the class of functions $g:[0, T] \times \mathbb{R} \times \mathbb{R} \rightarrow \mathbb{R}$ such that

(i) the map $t \rightarrow g(t, x, y)$ is measurable for each $x, y \in$ $\mathbb{R}$,

(ii) the map $x \rightarrow g(t, x, y)$ is continuous for each $x \in \mathbb{R}$,

(iii) the map $y \rightarrow g(t, x, y)$ is continuous for each $y \in \mathbb{R}$. [9].

We need the following definitions which can be found in

Definition 1. The Riemann-Liouville fractional integral of order $\alpha>0$ of function $f \in L^{1}\left(R^{+}\right)$is defined as

$$
I^{\alpha} f(t)=\frac{1}{\Gamma(\alpha)} \int_{0}^{t}(t-s)^{\alpha-1} f(s) d s,
$$

provided that the right side is pointwise defined on $(0, \infty)$.

Definition 2. Let $\alpha$ be a positive real number, such that $m-1 \leq$ $\alpha<m, m \in \mathbb{N}$, and $f^{m}(x)$ exists, a function of class $C$. Then Caputo fractional derivative of $f$ is defined as

$$
{ }^{c} D^{\alpha} f(t)=\frac{1}{\Gamma(m-\alpha)} \int_{0}^{t}(t-s)^{m-\alpha-1} f^{m}(s) d s,
$$

provided that the right side is pointwise defined on $(0, \infty)$, where $m=[\alpha]+1$ and $[\alpha]$ represents the integer part of $\alpha$.
Definition 3 (see [29]). The mapping $F: X \times X \rightarrow X$ has a coupled fixed point $(x, y) \in X \times X$ if $F(x, y)=x$ and $F(y, x)=$ $y$.

Theorem 4 (see [17]). Let $S$ be a nonempty, closed, convex, and bounded subset of the Banach space $X$ and $\widetilde{S}=S \times S$. Suppose that $A: X \rightarrow X$ and $B: S \rightarrow X$ are two operators such that

$\left(C_{1}\right)$ there exists $\varphi_{A} \in \Phi$ such that, for all $x, y \in X$, one has

$$
\|A x-A y\| \leq \sigma \varphi_{A}(\|x-y\|),
$$

for some constant $\sigma>0$,

$\left(C_{2}\right)$ B is completely continuous,

$\left(C_{3}\right) x=A x+B y \Rightarrow x \in S$, for all $y \in S$.

Then the operator $T(x, y)=A x+B y$ has at least a coupled fixed point in $\widetilde{S}$ whenever $\sigma<1$.

Lemma 5. The following result holds for fractional differential equations:

$$
I^{\alpha}\left[{ }^{c} D^{\alpha} h(t)\right]=h(t)+c_{0}+c_{1} t+c_{2} t^{2}+\cdots+c_{m-1} t^{m-1},
$$

for arbitrary $c_{i} \in \mathbb{R}, i=0,1,2, \ldots, m-1$, where $m=[\alpha]+1$ and $[\alpha]$ represents the integer part of $\alpha$.

\section{Existence Results}

Let us set the following notations for convenience:

$$
\begin{aligned}
\lambda= & \left(1-\delta_{1}\right)\left(1-\delta_{2} \eta_{2}^{n-1}\right)+\left(1-\delta_{2}\right) \delta_{1} \eta_{1}^{n-1}, \\
F_{0} & =\frac{2}{|\lambda|}\left(|f(1,0)|+\frac{1}{2} \max _{t \in[0,1]}|f(t, 0)|\right. \\
& \left.+\max _{\eta_{1} \in[0,1]}\left|f\left(\eta_{1}, 0\right)\right|+\max _{\eta_{2} \in[0,1]}\left|f\left(\eta_{2}, 0\right)\right|\right) .
\end{aligned}
$$

For the forthcoming analysis, we assume that

$\left(A_{1}\right)$ the function $x \rightarrow x-f(t, x)$ is increasing in $\mathbb{R}$ for all $t \in[0,1]$

$\left(A_{2}\right)$ there exists $M \geq L>0$ such that

$$
\begin{aligned}
& |f(t, x(t))-f(t, y(t))| \leq \frac{L|x(t)-y(t)|}{8(M+|x(t)-y(t)|)}, \\
& \frac{\left|f\left(1, \delta_{2} x\left(\eta_{2}\right)\right)-f\left(1, \delta_{2} y\left(\eta_{2}\right)\right)\right|}{|\lambda|} \\
& \quad \leq \sup \left\{\frac{L \delta_{2}|x(t)-y(t)|}{16\left(M+\delta_{2}|x(t)-y(t)|\right)}\right\}
\end{aligned}
$$




$$
\begin{aligned}
& \frac{\left|f\left(\eta_{1}, x\left(\eta_{1}\right)\right)-f\left(\eta_{1}, y\left(\eta_{1}\right)\right)\right|}{|\lambda|} \\
& \quad \leq \sup \left\{\frac{L|x(t)-y(t)|}{16(M+|x(t)-y(t)|)}\right\}, \\
& \frac{\left|f\left(\eta_{2}, x\left(\eta_{2}\right)\right)-f\left(\eta_{2}, y\left(\eta_{2}\right)\right)\right|}{|\lambda|} \\
& \quad \leq \sup \left\{\frac{L|x(t)-y(t)|}{16(M+|x(t)-y(t)|)}\right\} ;
\end{aligned}
$$

$\left(A_{3}\right)$ there exists a continuous function $h \in C([0$, $1], \mathbb{R})$ such that

$$
g(t, x(t), y(t)) \leq h(t), \quad x, y \in \mathbb{R}, t \in[0,1] .
$$

Lemma 6. If $f(0, x(0))=0$ and $\left.\left(\partial^{i} f(t, x(t)) / \partial t^{i}\right)\right|_{t=0}=0$ for $i=1,2, \ldots,(n-2)$, then integral representation of the system (2) is given by

$$
\begin{aligned}
& x(t)=f(t, x(t))+\frac{1}{\lambda}\left\{\delta_{2} \delta_{1} \eta_{1}^{n-1} f\left(\eta_{2}, x\left(\eta_{2}\right)\right)\right. \\
& -\delta_{1} \eta_{1}^{n-1} f(1, x(1)) \\
& \left.+\delta_{1}\left(1-\delta_{2} \eta_{2}^{n-1}\right) f\left(\eta_{1}, x\left(\eta_{1}\right)\right)\right\} \\
& +\frac{1}{\lambda}\left\{\left(1-\delta_{1}\right) \delta_{2} f\left(\eta_{2}, x\left(\eta_{2}\right)\right)\right. \\
& \left.-\left(1-\delta_{1}\right) f(1, x(1))-\left(1-\delta_{2}\right) \delta_{1} f\left(\eta_{1}, x\left(\eta_{1}\right)\right)\right\} \\
& \cdot t^{n-1}+\frac{1}{\Gamma(p)} \int_{0}^{t}(t-s)^{p-1} g\left(s, y(s), I^{\alpha} y(s)\right) d s \\
& -\left\{\frac{\delta_{1} \eta_{1}^{n-1}}{\lambda}+\frac{\left(1-\delta_{1}\right) t^{n-1}}{\lambda}\right\} \frac{1}{\Gamma(p)} \\
& \cdot \int_{0}^{1}(1-s)^{p-1} g\left(s, y(s), I^{\alpha} y(s)\right) d s \\
& +\left\{\frac{\delta_{1}\left(1-\delta_{2} \eta_{2}^{n-1}\right)}{\lambda}-\frac{\left(1-\delta_{2}\right) \delta_{1} t^{n-1}}{\lambda}\right\} \frac{1}{\Gamma(p)} \\
& \cdot \int_{0}^{\eta_{1}}\left(\eta_{1}-s\right)^{p-1} g\left(s, y(s), I^{\alpha} y(s)\right) d s \\
& +\left\{\frac{\delta_{2} \delta_{1} \eta_{1}^{n-1}}{\lambda}+\frac{\left(1-\delta_{1}\right) \delta_{2} t^{n-1}}{\lambda}\right\} \frac{1}{\Gamma(p)} \\
& \cdot \int_{0}^{\eta_{2}}\left(\eta_{2}-s\right)^{p-1} g\left(s, y(s), I^{\alpha} y(s)\right) d s, \\
& y(t)=f(t, y(t))+\frac{1}{\lambda}\left\{\delta_{2} \delta_{1} \eta_{1}^{n-1} f\left(\eta_{2}, y\left(\eta_{2}\right)\right)\right. \\
& -\delta_{1} \eta_{1}^{n-1} f(1, y(1)) \\
& \left.+\delta_{1}\left(1-\delta_{2} \eta_{2}^{n-1}\right) f\left(\eta_{1}, y\left(\eta_{1}\right)\right)\right\} \\
& +\frac{1}{\lambda}\left\{\left(1-\delta_{1}\right) \delta_{2} f\left(\eta_{2}, y\left(\eta_{2}\right)\right)\right.
\end{aligned}
$$

$$
\begin{aligned}
& \left.-\left(1-\delta_{1}\right) f(1, y(1))-\left(1-\delta_{2}\right) \delta_{1} f\left(\eta_{1}, y\left(\eta_{1}\right)\right)\right\} \\
& \cdot t^{n-1}+\frac{1}{\Gamma(p)} \int_{0}^{t}(t-s)^{p-1} g\left(s, x(s), I^{\alpha} x(s)\right) d s \\
& -\left\{\frac{\delta_{1} \eta_{1}^{n-1}}{\lambda}+\frac{\left(1-\delta_{1}\right) t^{n-1}}{\lambda}\right\} \frac{1}{\Gamma(p)} \\
& \cdot \int_{0}^{1}(1-s)^{p-1} g\left(s, x(s), I^{\alpha} x(s)\right) d s \\
& +\left\{\frac{\delta_{1}\left(1-\delta_{2} \eta_{2}^{n-1}\right)}{\lambda}-\frac{\left(1-\delta_{2}\right) \delta_{1} t^{n-1}}{\lambda}\right\} \frac{1}{\Gamma(p)} \\
& \cdot \int_{0}^{\eta_{1}}\left(\eta_{1}-s\right)^{p-1} g\left(s, x(s), I^{\alpha} x(s)\right) d s \\
& +\left\{\frac{\delta_{2} \delta_{1} \eta_{1}^{n-1}}{\lambda}+\frac{\left(1-\delta_{1}\right) \delta_{2} t^{n-1}}{\lambda}\right\} \frac{1}{\Gamma(p)} \\
& \cdot \int_{0}^{\eta_{2}}\left(\eta_{2}-s\right)^{p-1} g\left(s, x(s), I^{\alpha} x(s)\right) d s .
\end{aligned}
$$

Proof. Applying the operator $I^{p}$ on the first equation of system (2) and using Lemma 5, we obtain

$$
\begin{aligned}
x(t)= & f(t, x(t)) \\
& +\frac{1}{\Gamma(p)} \int_{0}^{t}(t-s)^{p-1} g\left(s, y(s), I^{\alpha} y(s)\right) d s \\
& +C_{0}+C_{1} t+C_{2} t^{2}+\cdots+C_{n-1} t^{n-1} .
\end{aligned}
$$

Applying the initial conditions $x^{(i)}(0)=0$, for $i=$ $1,2,3, \ldots,(n-2)$, we conclude that $C_{1}=C_{2}=\cdots=C_{n-2}=0$. Therefore (13) becomes

$$
\begin{aligned}
& x(t)=f(t, x(t))+\frac{1}{\Gamma(p)} \\
& \cdot \int_{0}^{t}(t-s)^{p-1} g\left(s, y(s), I^{\alpha} y(s)\right) d s+C_{0} \\
& \quad+C_{n-1} t^{n-1} .
\end{aligned}
$$

Now, to find the values of $C_{0}$ and $C_{n-1}$, since $x(0)=\delta_{1} x\left(\eta_{1}\right)$, from (14) we have

$$
\begin{aligned}
& f(0, x(0))+I^{p} g\left(0, y(0), I^{\alpha} y(0)\right)+C_{0} \\
& \quad=\delta_{1}\left\{f\left(\eta_{1}, x\left(\eta_{1}\right)\right)+\frac{1}{\Gamma(p)}\right. \\
& \quad \cdot \int_{0}^{\eta_{1}}\left(\eta_{1}-s\right)^{p-1} g\left(s, y(s), I^{\alpha} y(s)\right) d s+C_{0} \\
& \left.\quad+C_{n-1} \eta_{1}^{n-1}\right\} \Longrightarrow
\end{aligned}
$$




$$
\begin{gathered}
\left(1-\delta_{1}\right) C_{0}-\delta_{1} C_{n-1} \eta_{1}^{n-1}-\delta_{1}\left\{f\left(\eta_{1}, x\left(\eta_{1}\right)\right)+\frac{1}{\Gamma(p)}\right. \\
\left.\cdot \int_{0}^{\eta_{1}}\left(\eta_{1}-s\right)^{p-1} g\left(s, y(s), I^{\alpha} y(s)\right) d s\right\}=0 .
\end{gathered}
$$

Also, since $x(1)=\delta_{2} x\left(\eta_{2}\right)$, from (14), we have

$$
\begin{aligned}
& f(1, x(1))+\frac{1}{\Gamma(p)} \\
& \cdot \int_{0}^{1}(1-s)^{p-1} g\left(s, y(s), I^{\alpha} y(s)\right) d s+C_{0} \\
& +C_{n-1}=\delta_{2}\left\{f\left(\eta_{2}, x\left(\eta_{2}\right)\right)\right. \\
& \quad+\frac{1}{\Gamma(p)} \int_{0}^{\eta_{2}}\left(\eta_{2}-s\right)^{p-1} g\left(s, y(s), I^{\alpha} y(s)\right) d s+C_{0} \\
& \left.\quad+C_{n-1} \eta_{2}^{n-1}\right\} \Longrightarrow \\
& \left(1-\delta_{2}\right) C_{0}+\left(1-\delta_{2} \eta_{2}^{n-1}\right) C_{n-1}+f(1, x(1))+\frac{1}{\Gamma(p)} \\
& \quad \cdot \int_{0}^{1}(1-s)^{p-1} g\left(s, y(s), I^{\alpha} y(s)\right) d s \\
& \left.\quad+\frac{1}{\Gamma(p)} \int_{0}^{\eta_{2}}\left(\eta_{2}-s\right)^{p-1} g\left(s, y(s), I^{\alpha} y(s)\right) d s\right\} \\
& \quad 0\left(\eta_{2}, x\left(\eta_{2}\right)\right)
\end{aligned}
$$

Solving (15) and (16) for $C_{0}$, we get

$$
\begin{aligned}
& \left\{\left(1-\delta_{2} \eta_{2}^{n-1}\right)\left(1-\delta_{1}\right)+\delta_{1} \eta_{1}^{n-1}\left(1-\delta_{2}\right)\right\} C_{0} \\
& +\delta_{1} \eta_{1}^{n-1}\{f(1, x(1)) \\
& \left.+\frac{1}{\Gamma(p)} \int_{0}^{1}(1-s)^{p-1} g\left(s, y(s), I^{\alpha} y(s)\right) d s\right\} \\
& -\delta_{1}\left(1-\delta_{2} \eta_{2}^{n-1}\right)\left\{f\left(\eta_{1}, x\left(\eta_{1}\right)\right)\right. \\
& \left.+\frac{1}{\Gamma(p)} \int_{0}^{\eta_{1}}\left(\eta_{1}-s\right)^{p-1} g\left(s, y(s), I^{\alpha} y(s)\right) d s\right\} \\
& \quad-\delta_{2} \delta_{1} \eta_{1}^{n-1}\left\{f\left(\eta_{2}, x\left(\eta_{2}\right)\right)\right. \\
& \left.+\frac{1}{\Gamma(p)} \int_{0}^{\eta_{2}}\left(\eta_{2}-s\right)^{p-1} g\left(s, y(s), I^{\alpha} y(s)\right) d s\right\} \\
& \quad=0
\end{aligned}
$$

and using (7), we can write

$$
C_{0}=\frac{\delta_{1}\left(1-\delta_{2} \eta_{2}^{n-1}\right)}{\lambda}\left\{f\left(\eta_{1}, x\left(\eta_{1}\right)\right)\right.
$$

$$
\begin{aligned}
& \left.+\frac{1}{\Gamma(p)} \int_{0}^{\eta_{1}}\left(\eta_{1}-s\right)^{p-1} g\left(s, y(s), I^{\alpha} y(s)\right) d s\right\} \\
& +\frac{\delta_{2} \delta_{1} \eta_{1}^{n-1}}{\lambda}\left\{f\left(\eta_{2}, x\left(\eta_{2}\right)\right)\right. \\
& \left.+\frac{1}{\Gamma(p)} \int_{0}^{\eta_{2}}\left(\eta_{2}-s\right)^{p-1} g\left(s, y(s), I^{\alpha} y(s)\right) d s\right\} \\
& -\frac{\delta_{1} \eta_{1}^{n-1}}{\lambda}\{f(1, x(1)) \\
& \left.+\frac{1}{\Gamma(p)} \int_{0}^{1}(1-s)^{p-1} g\left(s, y(s), I^{\alpha} y(s)\right) d s\right\} .
\end{aligned}
$$

Similarly, solving (15) and (16) and using (7), we obtained that

$$
\begin{aligned}
C_{n-1} & =-\frac{\left(1-\delta_{1}\right)}{\lambda}\{f(1, x(1)) \\
& \left.+\frac{1}{\Gamma(p)} \int_{0}^{1}(1-s)^{p-1} g\left(s, y(s), I^{\alpha} y(s)\right) d s\right\} \\
& -\frac{\left(1-\delta_{2}\right) \delta_{1}}{\lambda}\left\{f\left(\eta_{1}, x\left(\eta_{1}\right)\right)\right. \\
& \left.+\frac{1}{\Gamma(p)} \int_{0}^{\eta_{1}}\left(\eta_{1}-s\right)^{p-1} g\left(s, y(s), I^{\alpha} y(s)\right) d s\right\} \\
+ & \frac{\left(1-\delta_{1}\right) \delta_{2}}{\lambda}\left\{f\left(\eta_{2}, x\left(\eta_{2}\right)\right)\right. \\
+ & \left.\frac{1}{\Gamma(p)} \int_{0}^{\eta_{2}}\left(\eta_{2}-s\right)^{p-1} g\left(s, y(s), I^{\alpha} y(s)\right) d s\right\} .
\end{aligned}
$$

Substituting the values of $C_{0}$ and $C_{1}$ in (14), we can write

$$
\begin{aligned}
& x(t)=f(t, x(t))+\frac{1}{\Gamma(p)} \\
& \cdot \int_{0}^{t}(t-s)^{p-1} g\left(s, y(s), I^{\alpha} y(s)\right) d s \\
& +\frac{\delta_{1}\left(1-\delta_{2} \eta_{2}^{n-1}\right)}{\lambda}\left\{f\left(\eta_{1}, x\left(\eta_{1}\right)\right)\right. \\
& \left.+\frac{1}{\Gamma(p)} \int_{0}^{\eta_{1}}\left(\eta_{1}-s\right)^{p-1} g\left(s, y(s), I^{\alpha} y(s)\right) d s\right\} \\
& +\frac{\delta_{2} \delta_{1} \eta_{1}^{n-1}}{\lambda}\left\{f\left(\eta_{2}, x\left(\eta_{2}\right)\right)\right.
\end{aligned}
$$




$$
\begin{aligned}
& \left.+\frac{1}{\Gamma(p)} \int_{0}^{\eta_{2}}\left(\eta_{2}-s\right)^{p-1} g\left(s, y(s), I^{\alpha} y(s)\right) d s\right\} \\
& -\frac{\delta_{1} \eta_{1}^{n-1}}{\lambda}\{f(1, x(1)) \\
& \left.+\frac{1}{\Gamma(p)} \int_{0}^{1}(1-s)^{p-1} g\left(s, y(s), I^{\alpha} y(s)\right) d s\right\} \\
& -\frac{\left(1-\delta_{1}\right)}{\lambda}\{f(1, x(1)) \\
& \left.+\frac{1}{\Gamma(p)} \int_{0}^{1}(1-s)^{p-1} g\left(s, y(s), I^{\alpha} y(s)\right) d s\right\} t^{n-1} \\
& -\frac{\left(1-\delta_{2}\right) \delta_{1}}{\lambda}\left\{f\left(\eta_{1}, x\left(\eta_{1}\right)\right)\right. \\
& \left.+t^{n-1}, \quad \frac{1}{\Gamma(p)} \int_{0}^{\eta_{2}}\left(\eta_{2}-s\right)^{p-1} g\left(s, y(s), I^{\alpha} y(s)\right) d s\right\} \\
& +t^{n-1}+\frac{\left(1-\delta_{1}\right) \delta_{2}}{\Gamma(p)}\left\{f\left(\eta_{2}, x\left(\eta_{2}\right)\right)\right. \\
& \left.+s)^{p-1} g\left(s, y(s), I^{\alpha} y(s)\right) d s\right\}
\end{aligned}
$$

which implies that

$$
\begin{aligned}
x( & t)=f(t, x(t))+\frac{1}{\lambda}\left\{\delta_{2} \delta_{1} \eta_{1}^{n-1} f\left(\eta_{2}, x\left(\eta_{2}\right)\right)\right. \\
& -\delta_{1} \eta_{1}^{n-1} f(1, x(1)) \\
& \left.+\delta_{1}\left(1-\delta_{2} \eta_{2}^{n-1}\right) f\left(\eta_{1}, x\left(\eta_{1}\right)\right)\right\} \\
& +\frac{1}{\lambda}\left\{\left(1-\delta_{1}\right) \delta_{2} f\left(\eta_{2}, x\left(\eta_{2}\right)\right)\right. \\
& \left.-\left(1-\delta_{1}\right) f(1, x(1))-\left(1-\delta_{2}\right) \delta_{1} f\left(\eta_{1}, x\left(\eta_{1}\right)\right)\right\} \\
& \cdot t^{n-1}+\frac{1}{\Gamma(p)} \int_{0}^{t}(t-s)^{p-1} g\left(s, y(s), I^{\alpha} y(s)\right) d s \\
& -\left\{\frac{\delta_{1} \eta_{1}^{n-1}}{\lambda}+\frac{\left(1-\delta_{1}\right) t^{n-1}}{\lambda}\right\} \frac{1}{\Gamma(p)} \\
& \cdot \int_{0}^{1}(1-s)^{p-1} g\left(s, y(s), I^{\alpha} y(s)\right) d s \\
& +\left\{\frac{\delta_{1}\left(1-\delta_{2} \eta_{2}^{n-1}\right)}{\lambda}-\frac{\left(1-\delta_{2}\right) \delta_{1} t^{n-1}}{\lambda}\right\} \frac{1}{\Gamma(p)}
\end{aligned}
$$

$$
\begin{aligned}
& +\left\{\frac{\delta_{2} \delta_{1} \eta_{1}^{n-1}}{\lambda}+\frac{\left(1-\delta_{1}\right) \delta_{2} t^{n-1}}{\lambda}\right\} \frac{1}{\Gamma(p)} \\
& \cdot \int_{0}^{\eta_{2}}\left(\eta_{2}-s\right)^{p-1} g\left(s, y(s), I^{\alpha} y(s)\right) d s .
\end{aligned}
$$

Similarly, repeating the above process with the second equation of system (2), we obtain integral equation (12).

Now, we are in a position to present the existence theorem for the system (2).

Theorem 7. Assume that hypotheses $\left(A_{1}\right)-\left(A_{3}\right)$ hold. Then there exists a solution for coupled systems (2) of higher order hybrid FHDEs with three-point boundary conditions.

Proof. Set $X=C([0,1], \mathbb{R})$ and a subset $S$ of $X$ defined by

$$
\begin{aligned}
& S=\{x \in X:\|x\| \leq N\} \\
& \text { where } N \geq 7 L+F_{0}+\frac{\|h\|_{L^{1}}}{\Gamma(p+1)}\left\{1+\frac{2}{|\lambda|}\left(1+\eta_{1}^{p}+\eta_{2}^{p}\right)\right\} .
\end{aligned}
$$

Clearly $S$ is a closed, convex, and bounded subset of the Banach space $X$. Now, since $x(t)$ is a solution of the FHDEs system (2) if and only if $x(t)$ satisfies the system of integral equations in Lemma 6, to show the existence solution of system (2) it is enough to show the existence solution of the integral equations in Lemma 6. For this, define two operators $A: X \rightarrow X$ and $B: S \rightarrow X$ by

$$
\begin{aligned}
A x & (t)=f(t, x(t))+\frac{1}{\lambda}\left\{\delta_{2} \delta_{1} \eta_{1}^{n-1} f\left(\eta_{2}, x\left(\eta_{2}\right)\right)\right. \\
& -\delta_{1} \eta_{1}^{n-1} f(1, x(1)) \\
& \left.+\delta_{1}\left(1-\delta_{2} \eta_{2}^{n-1}\right) f\left(\eta_{1}, x\left(\eta_{1}\right)\right)\right\} \\
& +\frac{1}{\lambda}\left\{\left(1-\delta_{1}\right) \delta_{2} f\left(\eta_{2}, x\left(\eta_{2}\right)\right)\right. \\
& \left.-\left(1-\delta_{1}\right) f(1, x(1))-\left(1-\delta_{2}\right) \delta_{1} f\left(\eta_{1}, x\left(\eta_{1}\right)\right)\right\} \\
& \cdot t^{n-1}, \\
& +(t)=\frac{1}{\Gamma(p)} \int_{0}^{t}(t-s)^{p-1} g\left(s, x(s), I^{\alpha} x(s)\right) d s \\
& -\left\{\frac{\delta_{1} \eta_{1}^{n-1}}{\lambda}+\frac{\left(1-\delta_{1}\right) t^{n-1}}{\lambda}\right\} \frac{1}{\Gamma(p)} \\
& +\int_{0}^{1} \frac{(1-s)^{p-1} g\left(s, x(s), I^{\alpha} x(s)\right) d s}{\lambda} \frac{\left(\eta_{1}-s\right)^{p-1} g\left(s, x(s), I^{\alpha} x(s)\right) d s}{\lambda(p)} \\
& \left.\frac{\delta_{1}\left(1-\delta_{2} \eta_{2}^{n-1}\right)}{\lambda}-\frac{\left(1-\delta_{2}\right) \delta_{1} t^{n-1}}{\lambda}\right\} \frac{1}{\Gamma(p)}
\end{aligned}
$$




$$
\begin{aligned}
& +\left\{\frac{\delta_{2} \delta_{1} \eta_{1}^{n-1}}{\lambda}+\frac{\left(1-\delta_{1}\right) \delta_{2} t^{n-1}}{\lambda}\right\} \frac{1}{\Gamma(p)} \\
& \cdot \int_{0}^{\eta_{2}}\left(\eta_{2}-s\right)^{p-1} g\left(s, x(s), I^{\alpha} x(s)\right) d s .
\end{aligned}
$$

Then the operators form of system (2) is

$$
\begin{aligned}
& x(t)=A x(t)+B y(t), \quad t \in[0,1], \\
& y(t)=A y(t)+B x(t), \quad t \in[0,1] .
\end{aligned}
$$

We have to show that the operators $A$ and $B$ satisfy all the conditions of Theorem 4 . For this, let $x, y \in X$; then we have

$$
\begin{aligned}
& |A x(t)-A y(t)| \leq|f(t, x(t))-f(t, y(t))| \\
& +\mid \frac{\delta_{2} \delta_{1} \eta_{1}^{n-1} f\left(\eta_{2}, x\left(\eta_{2}\right)\right)-\delta_{1} \eta_{1}^{n-1} f(1, x(1))+\delta_{1}\left(1-\delta_{2} \eta_{2}^{n-1}\right) f\left(\eta_{1}, x\left(\eta_{1}\right)\right)}{\lambda} \\
& -\frac{\delta_{2} \delta_{1} \eta_{1}^{n-1} f\left(\eta_{2}, y\left(\eta_{2}\right)\right)-\delta_{1} \eta_{1}^{n-1} f(1, y(1))+\delta_{1}\left(1-\delta_{2} \eta_{2}^{n-1}\right) f\left(\eta_{1}, y\left(\eta_{1}\right)\right)}{\lambda} \\
& +\mid \frac{\left(1-\delta_{1}\right) \delta_{2} f\left(\eta_{2}, x\left(\eta_{2}\right)\right)-\left(1-\delta_{1}\right) f(1, x(1))-\left(1-\delta_{2}\right) \delta_{1} f\left(\eta_{1}, x\left(\eta_{1}\right)\right)}{\lambda} \\
& -\frac{\left(1-\delta_{1}\right) \delta_{2} f\left(\eta_{2}, y\left(\eta_{2}\right)\right)-\left(1-\delta_{1}\right) f(1, y(1))-\left(1-\delta_{2}\right) \delta_{1} f\left(\eta_{1}, y\left(\eta_{1}\right)\right)}{\lambda}|| t^{n-1}|\leq| f(t, x(t))-f(t, y(t)) \mid \\
& +\delta_{1} \eta_{1}^{n-1}\left|\frac{f\left(1, \delta_{2} x\left(\eta_{2}\right)\right)-f\left(1, \delta_{2} y\left(\eta_{2}\right)\right)}{\lambda}\right|+\delta_{1}\left(1-\delta_{2} \eta_{2}^{n-1}\right)\left|\frac{f\left(\eta_{1}, x\left(\eta_{1}\right)\right)-f\left(\eta_{1}, y\left(\eta_{1}\right)\right)}{\lambda}\right| \\
& +\delta_{2} \delta_{1} \eta_{1}^{n-1}\left|\frac{f\left(\eta_{2}, x\left(\eta_{2}\right)\right)-f\left(\eta_{2}, y\left(\eta_{2}\right)\right)}{\lambda}\right|+\left(1-\delta_{1}\right)\left|\frac{f\left(1, \delta_{2} x\left(\eta_{2}\right)\right)-f\left(1, \delta_{2} y\left(\eta_{2}\right)\right)}{\lambda}\right|+\left(1-\delta_{2}\right) \\
& \cdot \delta_{1}\left|\frac{f\left(\eta_{1}, x\left(\eta_{1}\right)\right)-f\left(\eta_{1}, y\left(\eta_{1}\right)\right)}{\lambda}\right|+\left(1-\delta_{1}\right) \delta_{2}\left|\frac{f\left(\eta_{2}, x\left(\eta_{2}\right)\right)-f\left(\eta_{2}, y\left(\eta_{2}\right)\right)}{\lambda}\right|<|f(t, x(t))-f(t, y(t))| \\
& +\frac{2\left|f\left(1, \delta_{2} x\left(\eta_{2}\right)\right)-f\left(1, \delta_{2} y\left(\eta_{2}\right)\right)\right|}{|\lambda|}+\frac{2\left|f\left(\eta_{1}, x\left(\eta_{1}\right)\right)-f\left(\eta_{1}, y\left(\eta_{1}\right)\right)\right|}{|\lambda|}+\frac{2\left|f\left(\eta_{2}, x\left(\eta_{2}\right)\right)-f\left(\eta_{2}, y\left(\eta_{2}\right)\right)\right|}{|\lambda|}
\end{aligned}
$$

Taking the supremum over $t$ and using (7), we get

$$
\begin{aligned}
& \|A x(t)-A y(t)\| \leq \frac{1}{2}\left\{\frac{L\|x(t)-y(t)\|}{4(M+\|x(t)-y(t)\|)}\right. \\
& +\frac{L \delta_{2}\|x(t)-y(t)\|}{4\left(M+\delta_{2}\|x(t)-y(t)\|\right)} \\
& +\frac{L\|x(t)-y(t)\|}{4(M+\|x(t)-y(t)\|)} \\
& \left.+\frac{L\|x(t)-y(t)\|}{4(M+\|x(t)-y(t)\|)}\right\} \\
& =\frac{1}{2}\left\{\frac{3 L\|x(t)-y(t)\|}{4(M+\|x(t)-y(t)\|)}\right. \\
& \left.+\frac{L \delta_{2}\|x(t)-y(t)\|}{4\left(M+\delta_{2}\|x(t)-y(t)\|\right)}\right\} \leq \frac{1}{2} \\
& \cdot \varphi(\|x(t)-y(t)\|) .
\end{aligned}
$$

Thus $A$ satisfies condition $\left(C_{1}\right)$ of Theorem 4 with $\sigma=1 / 2$ and $\varphi(r)=3 L r / 4(M+r)+L \delta_{2} r / 4\left(M+\delta_{2} r\right)$.

Next, we show that $B$ is compact and continuous operator on $S$. Let $\left\{x_{m}\right\}$ be a sequence in $S$ such that $\left\{x_{m}\right\} \rightarrow x \in S$. Then for all $t \in[0,1]$, we have

$$
\begin{aligned}
& \lim _{m \rightarrow \infty} B x_{m}(t)=\frac{1}{\Gamma(p)} \\
& \cdot \lim _{m \rightarrow \infty} \int_{0}^{t}(t-s)^{p-1} g\left(s, x_{m}(s), I^{\alpha} x_{m}(s)\right) d s \\
& -\left\{\frac{\delta_{1} \eta_{1}^{n-1}}{\lambda}+\frac{\left(1-\delta_{1}\right) t^{n-1}}{\lambda}\right\} \lim _{m \rightarrow \infty} \frac{1}{\Gamma(p)} \\
& \cdot \int_{0}^{1}(1-s)^{p-1} g\left(s, x_{m}(s), I^{\alpha} x_{m}(s)\right) d s \\
& +\left\{\frac{\delta_{1}\left(1-\delta_{2} \eta_{2}^{n-1}\right)}{\lambda}-\frac{\left(1-\delta_{2}\right) \delta_{1} t^{n-1}}{\lambda}\right\} \lim _{m \rightarrow \infty} \frac{1}{\Gamma(p)}
\end{aligned}
$$


Discrete Dynamics in Nature and Society

7

$$
\begin{aligned}
& \cdot \int_{0}^{\eta_{1}}\left(\eta_{1}-s\right)^{p-1} g\left(s, x_{m}(s), I^{\alpha} x_{m}(s)\right) d s \\
& +\left\{\frac{\delta_{2} \delta_{1} \eta_{1}^{n-1}}{\lambda}+\frac{\left(1-\delta_{1}\right) \delta_{2} t^{n-1}}{\lambda}\right\} \lim _{m \rightarrow \infty} \frac{1}{\Gamma(p)} \\
& \cdot \int_{0}^{\eta_{2}}\left(\eta_{2}-s\right)^{p-1} g\left(s, x_{m}(s), I^{\alpha} x_{m}(s)\right) d s=\frac{1}{\Gamma(p)} \\
& \cdot \int_{0}^{t}(t-s)^{p-1} \lim _{m \rightarrow \infty} g\left(s, x_{m}(s), I^{\alpha} x_{m}(s)\right) d s \\
& -\left\{\frac{\delta_{1} \eta_{1}^{n-1}}{\lambda}+\frac{\left(1-\delta_{1}\right) t^{n-1}}{\lambda}\right\} \frac{1}{\Gamma(p)} \\
& \cdot \int_{0}^{1}(1-s)^{p-1} \lim _{m \rightarrow \infty} g\left(s, x_{m}(s), I^{\alpha} x_{m}(s)\right) d s \\
& +\left\{\frac{\delta_{1}\left(1-\delta_{2} \eta_{2}^{n-1}\right)}{\lambda}-\frac{\left(1-\delta_{2}\right) \delta_{1} t^{n-1}}{\lambda}\right\} \frac{1}{\Gamma(p)} \\
& \cdot \int_{0}^{\eta_{1}}\left(\eta_{1}-s\right)^{p-1} \lim _{m \rightarrow \infty} g\left(s, x_{m}(s), I^{\alpha} x_{m}(s)\right) d s \\
& +\left\{\frac{\delta_{2} \delta_{1} \eta_{1}^{n-1}}{\lambda}+\frac{\left(1-\delta_{1}\right) \delta_{2} t^{n-1}}{\lambda}\right\} \frac{1}{\Gamma(p)} \\
& \cdot \int_{0}^{\eta_{2}}\left(\eta_{2}-s\right)^{p-1} \lim _{m \rightarrow \infty} g\left(s, x_{m}(s), I^{\alpha} x_{m}(s)\right) d s \\
& =\frac{1}{\Gamma(p)} \int_{0}^{t}(t-s)^{p-1} g\left(s, x(s), I^{\alpha} x(s)\right) d s \\
& -\left\{\frac{\delta_{1} \eta_{1}^{n-1}}{\lambda}+\frac{\left(1-\delta_{1}\right) t^{n-1}}{\lambda}\right\} \frac{1}{\Gamma(p)} \\
& \cdot \int_{0}^{1}(1-s)^{p-1} g\left(s, x(s), I^{\alpha} x(s)\right) d s \\
& +\left\{\frac{\delta_{1}\left(1-\delta_{2} \eta_{2}^{n-1}\right)}{\lambda}-\frac{\left(1-\delta_{2}\right) \delta_{1} t^{n-1}}{\lambda}\right\} \frac{1}{\Gamma(p)} \\
& \cdot \int_{0}^{\eta_{1}}\left(\eta_{1}-s\right)^{p-1} g\left(s, x(s), I^{\alpha} x(s)\right) d s \\
& +\left\{\frac{\delta_{2} \delta_{1} \eta_{1}^{n-1}}{\lambda}+\frac{\left(1-\delta_{1}\right) \delta_{2} t^{n-1}}{\lambda}\right\} \frac{1}{\Gamma(p)} \\
& \cdot \int_{0}^{\eta_{2}}\left(\eta_{2}-s\right)^{p-1} g\left(s, x(s), I^{\alpha} x(s)\right) d s=B x(t) \text {. }
\end{aligned}
$$

Thus the map $B$ is continuous on $S$.

Let $x \in S$; then we have

$$
\begin{gathered}
|B x(t)| \leq \frac{1}{\Gamma(p)}\left|\int_{0}^{t}(t-s)^{p-1} g\left(s, x(s), I^{\alpha} x(s)\right) d s\right| \\
+\mid\left(\frac{\delta_{1} \eta_{1}^{n-1}}{\lambda}+\frac{\left(1-\delta_{1}\right) t^{n-1}}{\lambda}\right) \frac{1}{\Gamma(p)}
\end{gathered}
$$

$\cdot \int_{0}^{1}(1-s)^{p-1} g\left(s, x(s), I^{\alpha} x(s)\right) d s$

$+\mid\left(\frac{\delta_{1}\left(1-\delta_{2} \eta_{2}^{n-1}\right)}{\lambda}-\frac{\left(1-\delta_{2}\right) \delta_{1} t^{n-1}}{\lambda}\right) \frac{1}{\Gamma(p)}$

$\cdot \int_{0}^{\eta_{1}}\left(\eta_{1}-s\right)^{p-1} g\left(s, x(s), I^{\alpha} x(s)\right) d s \mid$
$+\mid\left(\frac{\delta_{2} \delta_{1} \eta_{1}^{n-1}}{\lambda}+\frac{\left(1-\delta_{1}\right) \delta_{2} t^{n-1}}{\lambda}\right) \frac{1}{\Gamma(p)}$

$\cdot \int_{0}^{\eta_{2}}\left(\eta_{2}-s\right)^{p-1} g\left(s, x(s), I^{\alpha} x(s)\right) d s \mid \leq \frac{1}{\Gamma(p)}$

$\cdot \int_{0}^{t}(t-s)^{p-1}\left|g\left(s, x(s), I^{\alpha} x(s)\right)\right| d s$

$+\left\{\frac{\left|\delta_{1} \eta_{1}^{n-1}\right|}{|\lambda|}+\frac{\left|\left(1-\delta_{1}\right) t^{n-1}\right|}{|\lambda|}\right\} \frac{1}{\Gamma(p)} \int_{0}^{1}(1-s)^{p-1}$

$\cdot\left|g\left(s, x(s), I^{\alpha} x(s)\right)\right| d s$

$+\left\{\frac{\left|\delta_{1}\left(1-\delta_{2} \eta_{2}^{n-1}\right)\right|}{|\lambda|}+\frac{\left|\left(1-\delta_{2}\right) \delta_{1} t^{n-1}\right|}{|\lambda|}\right\} \frac{1}{\Gamma(p)}$

$\cdot \int_{0}^{\eta_{1}}\left(\eta_{1}-s\right)^{p-1}\left|g\left(s, x(s), I^{\alpha} x(s)\right)\right| d s$

$+\left\{\frac{\left|\delta_{2} \delta_{1} \eta_{1}^{n-1}\right|}{|\lambda|}+\frac{\left|\left(1-\delta_{1}\right) \delta_{2} t^{n-1}\right|}{|\lambda|}\right\} \frac{1}{\Gamma(p)} \int_{0}^{\eta_{2}}\left(\eta_{2}\right.$

$-s)^{p-1}\left|g\left(s, x(s), I^{\alpha} x(s)\right)\right| d s \leq \frac{1}{\Gamma(p)} \int_{0}^{t}(t$

$-s)^{p-1}\|h(s)\| d s$

$+\frac{2}{|\lambda| \Gamma(p)}\left\{\int_{0}^{1}(1-s)^{p-1}\|h(s)\| d s\right.$

$+\int_{0}^{\eta_{1}}\left(\eta_{1}-s\right)^{p-1}\|h(s)\| d s$

$\left.+\int_{0}^{\eta_{2}}\left(\eta_{2}-s\right)^{p-1}\|h(s)\| d s\right\}$

$\leq \frac{\|h\|_{L^{1}}}{\Gamma(p)}\left\{\int_{0}^{t}(t-s)^{p-1} d s+\frac{2}{|\lambda|}\left(\int_{0}^{1}(1-s)^{p-1} d s\right.\right.$

$\left.\left.+\int_{0}^{\eta_{1}}\left(\eta_{1}-s\right)^{p-1} d s+\int_{0}^{\eta_{2}}\left(\eta_{2}-s\right)^{p-1} d s\right)\right\}$

$=\frac{\|h\|_{L^{1}}}{\Gamma(p)}\left\{\left.\frac{(t-s)^{p}}{-p}\right|_{0} ^{t}+\frac{2}{|\lambda|}\left(\left.\frac{(1-s)^{p}}{-p}\right|_{0} ^{1}\right.\right.$

$\left.\left.+\left.\frac{\left(\eta_{1}-s\right)^{p}}{-p}\right|_{0} ^{\eta_{1}}+\left.\frac{\left(\eta_{2}-s\right)^{p}}{-p}\right|_{0} ^{\eta_{2}}\right)\right\}=\frac{\|h\|_{L^{1}}}{\Gamma(p)}\left\{\frac{t^{p}}{p}\right.$ 


$$
\begin{aligned}
& \left.+\frac{2}{|\lambda|}\left(\frac{1}{p}+\frac{\eta_{1}^{p}}{p}+\frac{\eta_{2}^{p}}{p}\right)\right\}=\frac{\|h\|_{L^{1}}}{\Gamma(p+1)}\left\{t^{p}+\frac{2}{|\lambda|}(1\right. \\
& \left.\left.+\eta_{1}^{p}+\eta_{2}^{p}\right)\right\} \leq \frac{\|h\|_{L^{1}}}{\Gamma(p+1)}\left\{1+\frac{2}{|\lambda|}\left(1+\eta_{1}^{p}+\eta_{2}^{p}\right)\right\} .
\end{aligned}
$$

Taking the supremum over $t$, we get

$$
\|B x\| \leq \frac{\|h\|_{L^{1}}}{\Gamma(p+1)}\left\{1+\frac{2}{|\lambda|}\left(1+\eta_{1}^{p}+\eta_{2}^{p}\right)\right\}, \quad \forall x \in S .
$$

Thus $B$ is uniformly bounded on $S$.

Now, let $t_{1}, t_{2} \in[0,1]$ such that $t_{1} \neq t_{2}$; then for any $x \in S$, we have

$$
\begin{aligned}
& \left|B x\left(t_{1}\right)-B x\left(t_{2}\right)\right|=\mid \frac{1}{\Gamma(p)} \\
& \cdot \int_{0}^{t_{1}}\left(t_{1}-s\right)^{p-1} g\left(s, x(s), I^{\alpha} x(s)\right) d s \\
& -\left\{\frac{\delta_{1} \eta_{1}^{n-1}}{\lambda}+\frac{\left(1-\delta_{1}\right) t_{1}^{n-1}}{\lambda}\right\} \frac{1}{\Gamma(p)} \\
& \cdot \int_{0}^{1}(1-s)^{p-1} g\left(s, x(s), I^{\alpha} x(s)\right) d s \\
& +\left\{\frac{\delta_{1}\left(1-\delta_{2} \eta_{2}^{n-1}\right)}{\lambda}-\frac{\left(1-\delta_{2}\right) \delta_{1} t_{1}^{n-1}}{\lambda}\right\} \frac{1}{\Gamma(p)} \\
& \cdot \int_{0}^{\eta_{1}}\left(\eta_{1}-s\right)^{p-1} g\left(s, x(s), I^{\alpha} x(s)\right) d s \\
& +\left\{\frac{\delta_{2} \delta_{1} \eta_{1}^{n-1}}{\lambda}+\frac{\left(1-\delta_{1}\right) \delta_{2} t_{1}^{n-1}}{\lambda}\right\} \frac{1}{\Gamma(p)} \\
& \cdot \int_{0}^{\eta_{2}}\left(\eta_{2}-s\right)^{p-1} g\left(s, x(s), I^{\alpha} x(s)\right) d s-\frac{1}{\Gamma(p)} \\
& \cdot \int_{0}^{t_{2}}\left(t_{2}-s\right)^{p-1} g\left(s, x(s), I^{\alpha} x(s)\right) d s \\
& +\left\{\frac{\delta_{1} \eta_{1}^{n-1}}{\lambda}+\frac{\left(1-\delta_{1}\right) t_{1}^{n-1}}{\lambda}\right\} \frac{1}{\Gamma(p)} \\
& \cdot \int_{0}^{1}(1-s)^{p-1} g\left(s, x(s), I^{\alpha} x(s)\right) d s \\
& +\left\{\frac{\delta_{1}\left(1-\delta_{2} \eta_{2}^{n-1}\right)}{\lambda}-\frac{\left(1-\delta_{2}\right) \delta_{1} t_{1}^{n-1}}{\lambda}\right\} \frac{1}{\Gamma(p)} \\
& \cdot \int_{0}^{\eta_{1}}\left(\eta_{1}-s\right)^{p-1} g\left(s, x(s), I^{\alpha} x(s)\right) d s \\
& -\left\{\frac{\delta_{2} \delta_{1} \eta_{1}^{n-1}}{\lambda}+\frac{\left(1-\delta_{1}\right) \delta_{2} t_{1}^{n-1}}{\lambda}\right\} \frac{1}{\Gamma(p)}
\end{aligned}
$$

$$
\begin{aligned}
& \cdot \int_{0}^{\eta_{2}}\left(\eta_{2}-s\right)^{p-1} g\left(s, x(s), I^{\alpha} x(s)\right) d s \\
& \leq \frac{1}{\Gamma(p)} \mid \int_{0}^{t_{1}}\left(t_{1}-s\right)^{p-1} g\left(s, x(s), I^{\alpha} x(s)\right) d s \\
& -\int_{0}^{t_{2}}\left(t_{2}-s\right)^{p-1} g\left(s, x(s), I^{\alpha} x(s)\right) d s \\
& \leq \frac{1}{\Gamma(p)} \mid \int_{0}^{t_{1}}\left(t_{1}-s\right)^{p-1} h(s) d s \\
& -\int_{0}^{t_{1}}\left(t_{2}-s\right)^{p-1} h(s) d s+\int_{0}^{t_{1}}\left(t_{2}-s\right)^{p-1} h(s) d s \\
& -\int_{0}^{t_{2}}\left(t_{2}-s\right)^{p-1} h(s) d s \\
& \leq \frac{\|h\|_{L^{1}}}{\Gamma(p)}\left(\left|\int_{0}^{t_{1}}\left[\left(t_{1}-s\right)^{p-1}-\left(t_{2}-s\right)^{p-1}\right] d s\right|\right. \\
& \left.+\left|\int_{t_{1}}^{t_{2}}\left(t_{2}-s\right)^{p-1} d s\right|\right) \\
& =\frac{\|h\|_{L^{1}}}{\Gamma(p)}\left(\left|\frac{\left(t_{1}-s\right)^{p}}{-p}-\frac{\left(t_{2}-s\right)^{p}}{-p}\right|_{0}^{t_{1}}\right. \\
& \left.+\left|\frac{\left(t_{2}-s\right)^{p}}{-p}\right|_{t_{1}}^{t_{2}}\right)=\frac{\|h\|_{L^{1}}}{\Gamma(p)}\left(\left|\frac{\left(t_{2}-t_{1}\right)^{p}}{p}+\frac{t_{1}^{p}}{p}-\frac{t_{2}^{p}}{p}\right|\right. \\
& \left.+\left|\frac{\left(t_{2}-t_{1}\right)^{p}}{p}\right|\right) \leq \frac{\|h\|_{L^{1}}}{\Gamma(p+1)}\left(\left|t_{2}-t_{1}\right|^{p}+\left|t_{1}^{p}-t_{2}^{p}\right|\right. \\
& \left.+\left|t_{2}-t_{1}\right|^{p}\right) \text {. }
\end{aligned}
$$

Since $t^{p}$ is uniformly continuous on $[0,1]$ for $n-1<p<n$, for any $\varepsilon>0$ we can find $\delta_{1}^{*}>0$ such that

$$
\left|t_{1}^{p}-t_{2}^{p}\right|<\frac{\Gamma(p+1)}{3\|h\|_{L^{1}}} \varepsilon, \quad \text { whenever }\left|t_{1}-t_{2}\right|<\delta_{1}^{*} .
$$

Let $\delta^{*}=\min \left\{\delta_{1}^{*},\left(\Gamma(p+1) / 3\|h\|_{L^{1}}\right)^{1 / p}\right\}$; then, we have

$$
\begin{gathered}
\left|B x\left(t_{1}\right)-B x\left(t_{2}\right)\right| \leq \frac{\|h\|_{L^{1}}}{\Gamma(p+1)}\left(\frac{\Gamma(p+1)}{3\|h\|_{L^{1}}} \varepsilon\right. \\
\left.+\frac{\Gamma(p+1)}{3\|h\|_{L^{1}}} \varepsilon+\frac{\Gamma(p+1)}{3\|h\|_{L^{1}}} \varepsilon\right)=\varepsilon,
\end{gathered}
$$

whenever $\left|t_{1}-t_{2}\right|<\delta^{*}$.

Thus $B(S)$ is equicontinuous and hence $B$ is completely continuous on $S$.

To prove hypothesis $\left(C_{3}\right)$ of Theorem 4 , let $x \in X$ and $y \in S$ such that $x=A x+B y$; using (7) and (8), we have 


$$
\begin{aligned}
|x(t)| \leq \mid & A x(t)|+| B x(t) \mid \\
= & |f(t, x(t))|+\left|\frac{\delta_{2} \delta_{1} \eta_{1}^{n-1} f\left(\eta_{2}, x\left(\eta_{2}\right)\right)-\delta_{1} \eta_{1}^{n-1} f(1, x(1))+\delta_{1}\left(1-\delta_{2} \eta_{2}^{n-1}\right) f\left(\eta_{1}, x\left(\eta_{1}\right)\right)}{\lambda}\right| \\
& +\left|\frac{\left(1-\delta_{1}\right) \delta_{2} f\left(\eta_{2}, x\left(\eta_{2}\right)\right)-\left(1-\delta_{1}\right) f(1, x(1))-\left(1-\delta_{2}\right) \delta_{1} f\left(\eta_{1}, x\left(\eta_{1}\right)\right)}{\lambda}\right| t^{n-1} \mid \\
& +\frac{1}{\Gamma(p)}\left|\int_{0}^{t}(t-s)^{p-1} g\left(s, x(s), I^{\alpha} x(s)\right) d s\right| \\
& +\left|\frac{\delta_{1} \eta_{1}^{n-1}}{\lambda}+\frac{\left(1-\delta_{1}\right) t^{n-1}}{\lambda}\right| \frac{1}{\Gamma(p)}\left|\int_{0}^{1}(1-s)^{p-1} g\left(s, x(s), I^{\alpha} x(s)\right) d s\right| \\
& +\left|\frac{\delta_{1}\left(1-\delta_{2} \eta_{2}^{n-1}\right)}{\lambda}-\frac{\left(1-\delta_{2}\right) \delta_{1} t^{n-1}}{\lambda}\right| \frac{1}{\Gamma(p)}\left|\int_{0}^{\eta_{1}}\left(\eta_{1}-s\right)^{p-1} g\left(s, x(s), I^{\alpha} x(s)\right) d s\right| \\
& +\left|\frac{\delta_{2} \delta_{1} \eta_{1}^{n-1}}{\lambda}+\frac{\left(1-\delta_{1}\right) \delta_{2} t^{n-1}}{\lambda}\right| \frac{1}{\Gamma(p)}\left|\int_{0}^{\eta_{2}}\left(\eta_{2}-s\right)^{p-1} g\left(s, x(s), I^{\alpha} x(s)\right) d s\right| \\
\leq & |f(t, x(t))|+\frac{\left|f\left(\eta_{2}, x\left(\eta_{2}\right)\right)+f(1, x(1))\right|+\left|f\left(\eta_{1}, x\left(\eta_{1}\right)\right)\right|}{|\lambda|}+\frac{\left|f\left(\eta_{2}, x\left(\eta_{2}\right)\right)+f(1, x(1))\right|+\left|f\left(\eta_{1}, x\left(\eta_{1}\right)\right)\right|}{|\lambda|} \\
& +\frac{\|h\|_{L^{1}}}{\Gamma(p+1)}\left\{1+\frac{2}{|\lambda|}\left(1+\eta_{1}^{p}+\eta_{2}^{p}\right)\right\} .
\end{aligned}
$$

This implies

$$
\begin{aligned}
& |x(t)| \leq|f(t, x(t))-f(t, 0)|+|f(t, 0)| \\
& +2\left(\frac{|f(1,0)|}{|\lambda|}+\frac{\left|f\left(\eta_{1}, 0\right)\right|}{|\lambda|}+\frac{\left|f\left(\eta_{2}, 0\right)\right|}{|\lambda|}\right) \\
& +2\left(\frac{\left|f\left(1, \delta_{2} x\left(\eta_{2}\right)\right)-f(1,0)\right|}{|\lambda|}\right. \\
& +\frac{\left|f\left(\eta_{1}, x\left(\eta_{1}\right)\right)-f\left(\eta_{1}, 0\right)\right|}{|\lambda|} \\
& \left.+\frac{\left|f\left(\eta_{2}, x\left(\eta_{2}\right)\right)-f\left(\eta_{2}, 0\right)\right|}{|\lambda|}\right)+\frac{\|h\|_{L^{1}}}{\Gamma(p+1)}\{1 \\
& \left.+\frac{2}{|\lambda|}\left(1+\eta_{1}^{p}+\eta_{2}^{p}\right)\right\} \leq L+2(L+L+L) \\
& +\frac{2}{|\lambda|}\left(\frac{1}{2}|f(t, 0)|+|f(1,0)|+\left|f\left(\eta_{1}, 0\right)\right|\right. \\
& \left.+\left|f\left(\eta_{2}, 0\right)\right|\right)+\frac{\|h\|_{L^{1}}}{\Gamma(p+1)}\{1 \\
& \left.+\frac{2}{|\lambda|}\left(1+\eta_{1}^{p}+\eta_{2}^{p}\right)\right\} \leq 7 L+F_{0}+\frac{\|h\|_{L^{1}}}{\Gamma(p+1)}\{1 \\
& \left.\left.+1+\eta_{1}^{p}+\eta_{2}^{p}\right)\right\} \cdot
\end{aligned}
$$

Taking the supremum over $t$ on $[0,1]$, we can write

$$
\begin{aligned}
\|x(t)\| \leq & 7 L+F_{0} \\
& +\frac{\|h\|_{L^{1}}}{\Gamma(p+1)}\left\{1+\frac{2}{|\lambda|}\left(1+\eta_{1}^{p}+\eta_{2}^{p}\right)\right\} \leq N .
\end{aligned}
$$

That is, $x \in S$. Thus condition $\left(C_{3}\right)$ of Theorem 4 holds. Therefore, all the conditions of Theorem 4 are satisfied; hence the operator $T(x, y)=A x+B y$ has a coupled fixed point on $\widetilde{\mathrm{S}}$. Consequently, system (2) has a solution defined on $[0,1]$.

To illustrate Theorem 7 , we construct the following example.

Example 8. We discuss the following hybrid fractional differential equations with three-point boundary conditions:

$$
\begin{aligned}
& D^{5 / 2}\left[x(t)-\frac{e^{-t}|x(t)|}{11+|x(t)|}\right] \\
& =\frac{t^{3}}{3}-\left(\cos |y(t)|+\cos \left|I^{3 / 2} y(t)\right|\right), \\
& D^{5 / 2}\left[y(t)-\frac{e^{-t}|y(t)|}{11+|y(t)|}\right] \\
& =\frac{t^{3}}{3}-\left(\cos |x(t)|+\cos \left|I^{3 / 2} x(t)\right|\right),
\end{aligned}
$$




$$
\begin{aligned}
& x(0)=\frac{1}{4} x\left(\frac{1}{4}\right), \\
& y(0)=\frac{1}{4} y\left(\frac{1}{4}\right), \\
& x(1)=\frac{1}{2} x\left(\frac{1}{2}\right), \\
& y(1)=\frac{1}{2} y\left(\frac{1}{2}\right), \\
& x^{\prime}(0)=y^{\prime}(0)=0,
\end{aligned}
$$

where $t \in[0,1], f:[0,1] \times \mathbb{R} \rightarrow \mathbb{R}, f(0, x(0))=0$, and $g:[0,1] \times \mathbb{R} \times \mathbb{R} \rightarrow \mathbb{R} \cdot D^{5 / 2}$ is the Caputo fractional derivative of order $5 / 2$.

Here

$$
\begin{aligned}
\delta_{1} & =\eta_{1}=\frac{1}{4}, \\
\delta_{2} & =\eta_{2}=\frac{1}{2}, \\
f(t, x(t)) & =\frac{e^{-t}|x(t)|}{11+|x(t)|}, \\
g\left(t, x(t), I^{\alpha} x(t)\right) & =\frac{t^{3}}{3} \\
& -\left(\cos |x(t)|+\cos \left|I^{3 / 2} x(t)\right|\right) .
\end{aligned}
$$

$$
\begin{aligned}
\lambda= & \frac{19}{32}, \\
F_{0} & =\frac{2}{|\lambda|}\left(|f(1,0)|+\frac{1}{2} \max _{t \in[0,1]}|f(t, 0)|\right. \\
& \left.+\max _{\eta_{1} \in[0,1]}\left|f\left(\eta_{1}, 0\right)\right|+\max _{\eta_{2} \in[0,1]}\left|f\left(\eta_{2}, 0\right)\right|\right)=0 .
\end{aligned}
$$

Now, for $M=11$ and $L=512 / 19 e<M$, we have

$$
\begin{aligned}
& |f(t, x(t))-f(t, y(t))|=\mid \frac{e^{-t}|x(t)|}{11+|x(t)|} \\
& \quad-\frac{e^{-t}|y(t)|}{11+|y(t)|}|\leq| \frac{|x(t)-y(t)|+|y(t)|}{11+|x(t)-y(t)|+|y(t)|} \\
& \quad-\frac{|y(t)|}{11+|x(t)-y(t)|+|y(t)|} \mid \\
& \quad \leq\left|\frac{|x(t)-y(t)|}{11+|x(t)-y(t)|+|y(t)|}\right| \\
& \quad \leq \frac{|x(t)-y(t)|}{11+|x(t)-y(t)|} \leq \frac{(512 / 19 e)|x(t)-y(t)|}{16(11+|x(t)-y(t)|)}
\end{aligned}
$$

$$
\begin{aligned}
& \frac{\left|f\left(1, \delta_{2} x\left(\eta_{2}\right)\right)-f\left(1, \delta_{2} y\left(\eta_{2}\right)\right)\right|}{|\lambda|} \\
& \quad=\frac{|f(1,(1 / 2) x(1 / 2))-f(1,(1 / 2) y(1 / 2))|}{19 / 32} \\
& \quad=\frac{32}{19}\left|\frac{e^{-1}|(1 / 2) x(1 / 2)|}{11+|(1 / 2) x(1 / 2)|}-\frac{e^{-1}|(1 / 2) y(1 / 2)|}{11+|(1 / 2) y(1 / 2)|}\right| \\
& \frac{\left|f\left(1, \delta_{2} x\left(\eta_{2}\right)\right)-f\left(1, \delta_{2} y\left(\eta_{2}\right)\right)\right|}{|\lambda|} \\
& \quad \leq \frac{32}{19 e} \mid \frac{\delta_{2}|x(1 / 2)-y(1 / 2)|+\delta_{2}|y(1 / 2)|}{11+\delta_{2}|x(1 / 2)-y(1 / 2)|+\delta_{2}|y(1 / 2)|} \\
& \quad-\frac{\delta_{2}|y(1 / 2)|}{11+\delta_{2}|x(1 / 2)-y(1 / 2)|+\delta_{2}|y(1 / 2)|} \mid \\
& \quad \leq \frac{32}{19 e} \mid \frac{32}{11+\delta_{2}|x(1 / 2)-y(1 / 2)|+\delta_{2}|y(1 / 2)|} \times \frac{\delta_{2}|x(1 / 2)-y(1 / 2)|}{11+\delta_{2}|x(1 / 2)-y(1 / 2)|} \\
& \quad \leq \sup \left\{\frac{(512 / 19 e) \delta_{2}|x(t)-y(t)|}{16\left(11+\delta_{2}|x(t)-y(t)|\right)} .\right.
\end{aligned}
$$

Similarly,

$$
\begin{aligned}
& \frac{\left|f\left(\eta_{1}, x\left(\eta_{1}\right)\right)-f\left(\eta_{1}, y\left(\eta_{1}\right)\right)\right|}{|\lambda|} \\
& \quad \leq \sup \left\{\frac{(512 / 19 e) \delta_{2}|x(t)-y(t)|}{16\left(11+\delta_{2}|x(t)-y(t)|\right)}\right\}, \\
& \frac{\left|f\left(\eta_{2}, x\left(\eta_{2}\right)\right)-f\left(\eta_{2}, y\left(\eta_{2}\right)\right)\right|}{|\lambda|} \\
& \quad \leq \sup \left\{\frac{(512 / 19 e) \delta_{2}|x(t)-y(t)|}{16\left(11+\delta_{2}|x(t)-y(t)|\right)}\right\} .
\end{aligned}
$$

Next,

$$
\begin{aligned}
g\left(t, x(t), I^{\alpha} x(t)\right)= & \frac{t^{3}}{3} \\
& -\left(\cos |x(t)|+\cos \left|I^{3 / 2} x(t)\right|\right) \\
\leq & \frac{t^{3}}{3}=h(t) .
\end{aligned}
$$

That is, there exists a continuous function $h \in C([0,1], \mathbb{R})$ such that

$$
g(t, x(t), y(t)) \leq h(t), \quad x, y \in \mathbb{R}, t \in[0,1]
$$


Finally, since

$$
\begin{aligned}
\|h(t)\| & =\sup \int_{0}^{1} \frac{t^{3}}{3} d t=\frac{1}{12}, \\
L & =\frac{512}{19 e} \\
F_{0} & =0 \\
\lambda & =\frac{19}{32}
\end{aligned}
$$

we can write

$$
7 L+F_{0}+\frac{\|h\|_{L^{1}}}{\Gamma(p+1)}\left\{1+\frac{2}{|\lambda|}\left(1+\eta_{1}^{p}+\eta_{2}^{p}\right)\right\}<70 .
$$

Thus $N \geq 70$. It follows that assumptions $\left(A_{1}\right)$ and $\left(A_{2}\right)$ are satisfied. Therefore, by Theorem 7 we conclude that problem (36) has a solution.

\section{Conclusion}

We have successfully developed appropriate conditions for existence of at least one solution to a complicated higher order coupled system of nonlinear hybrid fractional differential equations. The respective conditions have been derived by using coupled fixed point theorem of Krasnoselskii type. The obtained results were also demonstrated by a suitable example.

\section{Conflicts of Interest}

The authors declare that they have no conflicts of interest.

\section{Authors' Contributions}

All the authors have equal contribution. The first two authors designed the problem and the last two authors read and corrected its style and language and prepared the final version.

\section{Acknowledgments}

This work was financially supported by the Rajamangala University of Technology Thanyaburi (RMUTT), Pathum Thani, Thailand.

\section{References}

[1] D. Baleanu, A. C. J. Luo, and J. A. T. Machado, "Fractional dynamics and control," Fractional Dynamics and Control, pp. 1313, 2012.

[2] Z. Dahmani, M. M. Mesmoudi, and R. Bebbouchi, "The foam drainage equation with time- and space-fractional derivatives solved by the Adomian method," Electronic Journal of Qualitative Theory of Differential Equations, pp. 1-10, 2008.

[3] W. G. Glockle and T. F. Nonnenmacher, "A fractional calculus approach to self-similar protein dynamics," Biophysical Journal, vol. 68 , no. 1 , pp. 46-53, 1995.
[4] R. Hilfer, Applications of Fractional Calculus in Physics, World Scientific Publishing Co., Singapore, 2000.

[5] R. Metzler, W. Schick, H. Kilian, and T. F. Nonnenmacher, "Relaxation in filled polymers: A fractional calculus approach," The Journal of Chemical Physics, vol. 103, no. 16, pp. 7180-7186, 1995.

[6] J. Sabatier, O. P. Agrawal, and J. A. Tenreiro MacHado, "Preface," Advances in Fractional Calculus: Theoretical Developments and Applications in Physics and Engineering, pp. xi-xiii, 2007.

[7] K. Balachandran and J. Y. Park, "Nonlocal Cauchy problem for abstract fractional semilinear evolution equations," Nonlinear Analysis. Theory, Methods \& Applications. An International Multidisciplinary Journal, vol. 71, no. 10, pp. 4471-4475, 2009.

[8] O. K. Jaradat, A. Al-Omari, and S. Momani, "Existence of the mild solution for fractional semilinear initial value problems," Nonlinear Analysis. Theory, Methods \& Applications. An International Multidisciplinary Journal, vol. 69, no. 9, pp. 3153-3159, 2008.

[9] A. A. Kilbas, H. M. Srivastava, and J. J. Trujillo, "Preface," NorthHolland Mathematics Studies, vol. 204, no. C, pp. vii-x, 2006.

[10] B. N. Lundstrom, M. H. Higgs, W. J. Spain, and A. L. Fairhall, "Fractional differentiation by neocortical pyramidal neurons," Nature Neuroscience, vol. 11, no. 11, pp. 1335-1342, 2008.

[11] I. Podlubny, "Geometric and physical interpretation of fractional integration and fractional differentiation," Fractional Calculus and Applied Analysis, vol. 5, no. 4, pp. 367-386, 2002.

[12] S. G. Samko, A. A. Kilbas, and O. I. Marichev, Fractional Integrals and Derivatives, Theory and Applications, Gordon and Breach, Yverdon, Switzerland, 1993.

[13] B. Ahmad, S. K. Ntouyas, and A. Alsaedi, "Existence results for a system of coupled hybrid fractional differential equations," The Scientific World Journal, vol. 2014, Article ID 426438, 6 pages, 2014.

[14] A. Anber, S. Belarbi, and Z. Dahmani, "New existence and uniqueness results for fractional differential equations," Analele Ştiinţifice ale Universităţii Ovidius Constanţa. Seria Matematică, vol. 21, no. 3, pp. 33-41, 2013.

[15] A. Babakhani, "Existence of solution for a coupled system of fractional integro-differential equations on an unbounded domain," Analysis in Theory and Applications, vol. 29, no. 1, pp. 47-61, 2013.

[16] D. Baleanu, H. Khan, H. Jafari, R. A. Khan, and M. Alipour, "On existence results for solutions of a coupled system of hybrid boundary value problems with hybrid conditions," Advances in Difference Equations, vol. 2015, no. 1, article no. 318, 2015.

[17] T. Bashiri, S. M. Vaezpour, and C. Park, "A coupled fixed point theorem and application to fractional hybrid differential problems," Fixed Point Theory and Applications, vol. 2016, no. 1, article no. 23, 2016.

[18] B. Ahmad and J. J. Nieto, "Existence results for a coupled system of nonlinear fractional differential equations with threepoint boundary conditions," Computers \& Mathematics with Applications, vol. 58, no. 9, pp. 1838-1843, 2009.

[19] Y. Chen and H.-L. An, "Numerical solutions of coupled Burgers equations with time- and space-fractional derivatives," Applied Mathematics and Computation, vol. 200, no. 1, pp. 87-95, 2008.

[20] V. Gafiychuk, B. Datsko, and V. Meleshko, "Mathematical modeling of time fractional reaction-diffusion systems," Journal of Computational and Applied Mathematics, vol. 220, no. 1-2, pp. 215-225, 2008. 
[21] V. Gafiychuk, B. Datsko, V. Meleshko, and D. Blackmore, "Analysis of the solutions of coupled nonlinear fractional reactiondiffusion equations," Chaos, Solitons \& Fractals, vol. 41, no. 3, pp. 1095-1104, 2009.

[22] C. S. Goodrich, "Existence of a positive solution to systems of differential equations of fractional order," Computers \& Mathematics with Applications. An International Journal, vol. 62, no. 3, pp. 1251-1268, 2011.

[23] M. P. Lazarević, "Finite time stability analysis of PD $\alpha$-fractional control of robotic time-delay systems," Mechanics Research Communications, vol. 33, no. 2, pp. 269-279, 2006.

[24] S. K. Ntouyas and M. Obaid, "A coupled system of fractional differential equations with nonlocal integral boundary conditions," Advances in Difference Equations, vol. 2012, article no. 130, 2012.

[25] X. Su, "Boundary value problem for a coupled system of nonlinear fractional differential equations," Applied Mathematics Letters, vol. 22, no. 1, pp. 64-69, 2009.

[26] M. Benchohra, S. Hamani, and S. K. Ntouyas, "Boundary value problems for differential equations with fractional order and nonlocal conditions," Nonlinear Analysis. Theory, Methods \& Applications. An International Multidisciplinary Journal, vol. 71, no. 7-8, pp. 2391-2396, 2009.

[27] X. Liu and M. Jia, "Multiple solutions of nonlocal boundary value problems for fractional differential equations on the halfline," Electronic Journal of Qualitative Theory of Differential Equations, pp. 1-14, 2011.

[28] S. Zhang, "Existence of solution for a boundary value problem of fractional order," Acta Mathematica Scientia, vol. 26, no. 2, pp. 220-228, 2006.

[29] S. S. Chang, Y. J. Cho, and N. J. Huang, "Coupled fixed point theorems with applications," Journal of the Korean Mathematical Society, vol. 33, no. 3, pp. 575-585, 1996. 


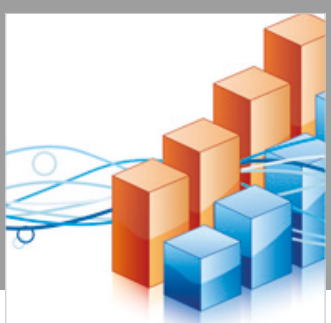

Advances in

Operations Research

\section{-n-m}
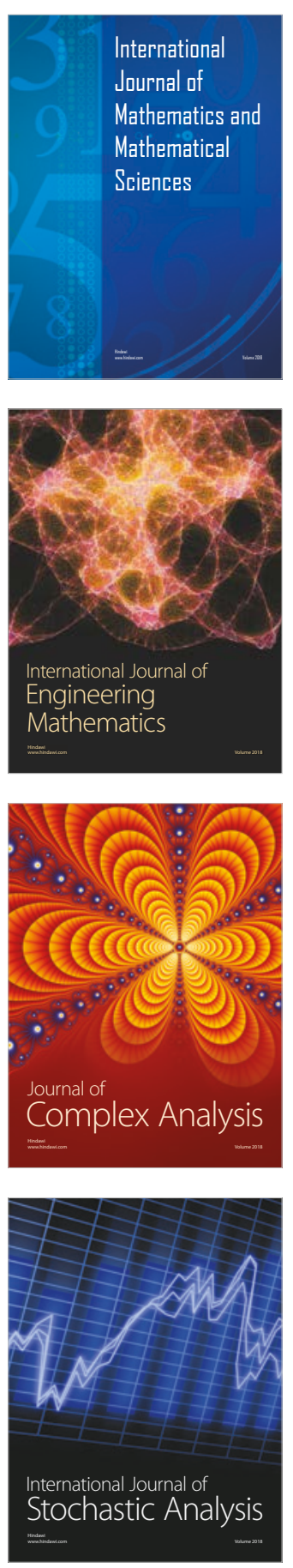
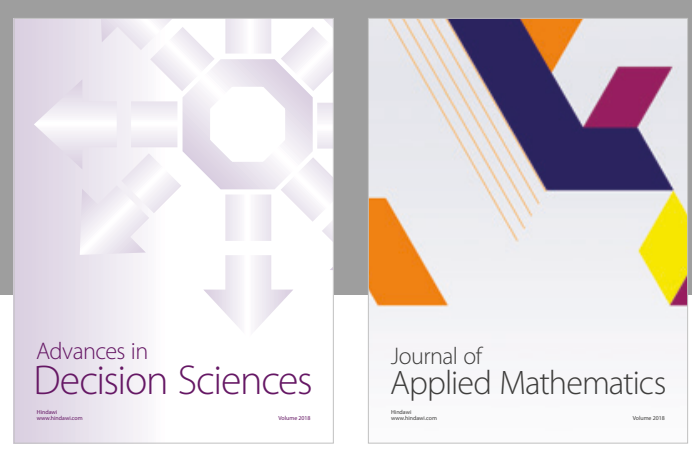

Journal of

Applied Mathematics
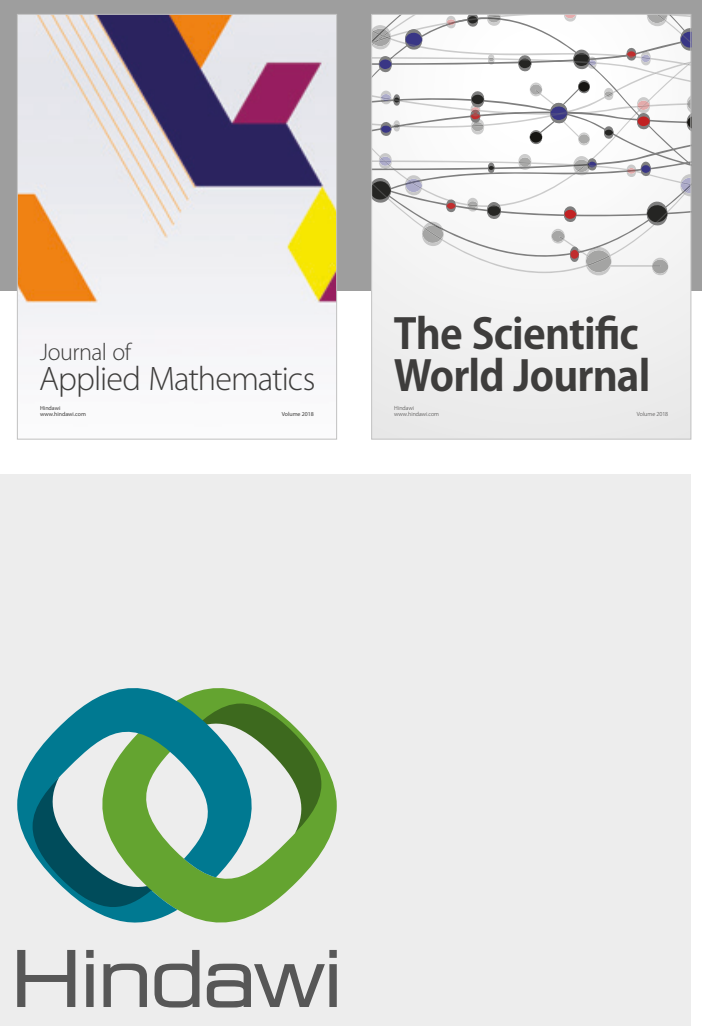

Submit your manuscripts at

www.hindawi.com

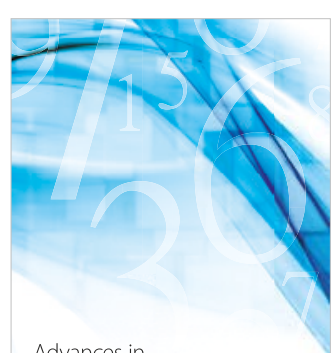

Advances in
Numerical Analysis
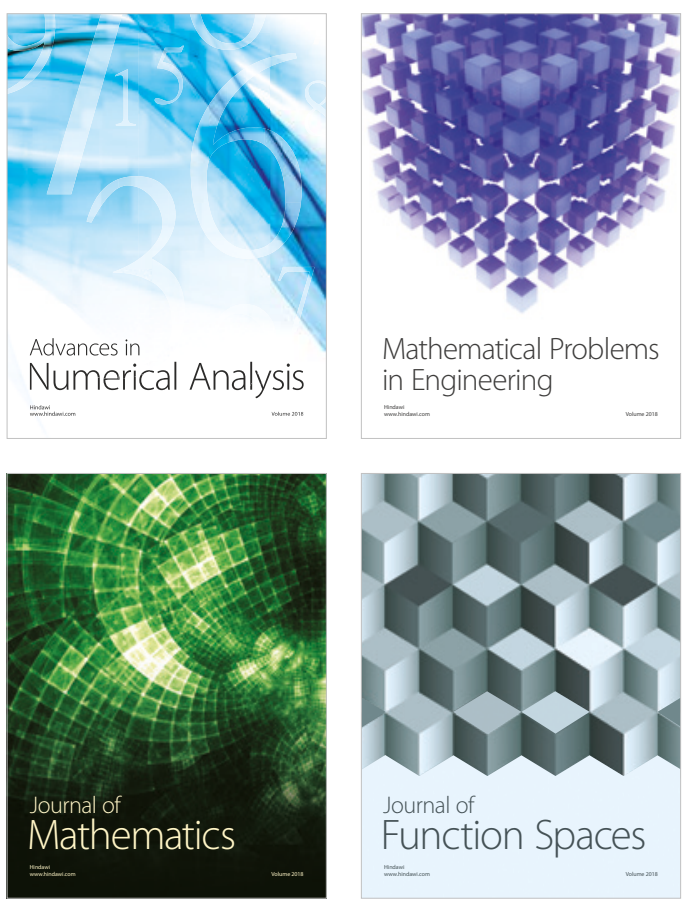

Mathematical Problems in Engineering

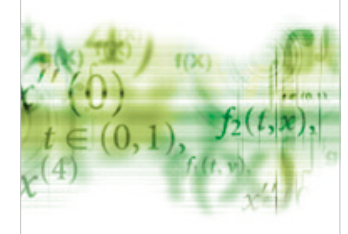

International Journal of

Differential Equations

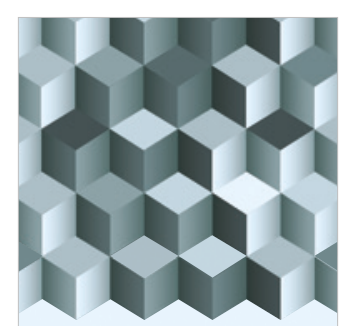

Journal of

Function Spaces

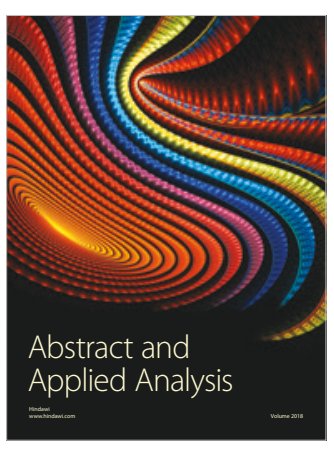

The Scientific

World Journal

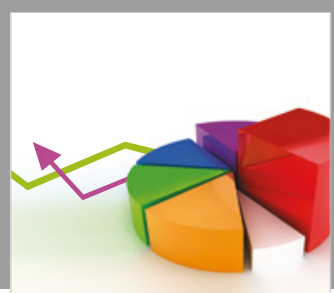

Journal of

Probability and Statistics
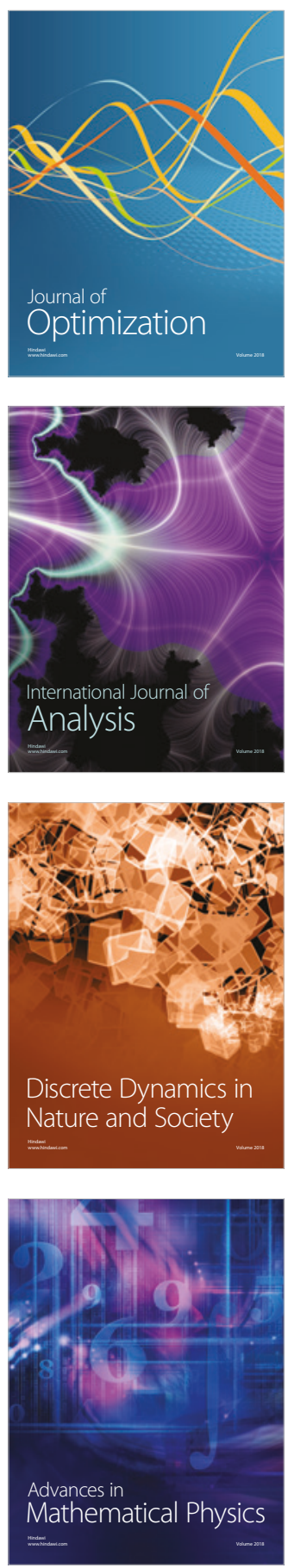